\title{
To T or not to B: germline $R U N X 1$ mutation preferences in pediatric ALL predisposition
}

\author{
Serine Avagyan ${ }^{1}$ and Anna L. Brown ${ }^{2,3,4}$ \\ 'Dana-Farber/Boston Children's Hospital Cancer and Blood Disorders Center, Boston, Massachusetts, USA. ²Department of Cenetics and Molecular Pathology, SA Pathology, and Centre for Cancer Biology, \\ SA Pathology and University of South Australia, Adelaide, South Australia, Australia. ${ }^{4}$ School of Medicine, University of Adelaide, Adelaide, South Australia, Australia.
}

\begin{abstract}
Germline RUNX1 variants have been identified in relation to myeloid malignancy predisposition, with lymphoid hematological malignancies present at a lower frequency in families. In this issue of the JCI, Li and Yang et al. examined the frequency and type of germline RUNX1 variants in pediatric patients with acute lymphoblastic leukemia (ALL). Patients with T cell ALL (T-ALL) harbored rare, damaging RUNX1 mutations that were not seen in patients with B cell ALL (B-ALL). Further, several of the T-ALL-associated RUNX1 variants had potential dominant-negative activity. RUNX1-mutated T-ALL cases were also associated with somatic JAK3 mutations and enriched for the early $T$ cell precursor (ETP) leukemia subtype, a finding that was validated when RUNX1 and JAK3 mutations were combined in mice. This study confirms germline RUNX1 predisposition beyond myeloid malignancy, demonstrates the importance of examining both germline and somatic mutations in malignancy cohorts, and demarcates the ETP ALL subtype as a flag for germline predisposition in patients.
\end{abstract}

\section{Germline predisposition to hematological malignancy}

Acute lymphoblastic leukemia (ALL) is the most common cancer in children and increasingly recognized as having an inherited component in some cases. Initially, ALL was annotated as part of more general cancer predisposition syndromes, such as germline TP53 mutations in patients manifesting as low-hypodiploid ALL in Li-Fraumeni syndrome (1). In recent years, syndromes with a predominant predisposition to hematological malignancies and in some cases exclusive predisposition to ALL (e.g., PAX5, IKZF1) have emerged (Figure 1A) (1). It has been more than 20 years since the first report of germline RUNX1 variants as causative for familial platelet disorder (RUNX1FPD), most commonly observed with myeloid malignancy (2). Germline RUNX1 variants are present in approximately $12 \%$ of families with inherited myelodysplastic syndrome(s) and acute myeloid leukemia and $3 \%$ of patients with inherited bone marrow failure syndromes, excluding Fanconi anemia $(3,4)$. Ongoing clinical and research studies have identified several hundred families (5) and broadened the range of hematological malignancies in the germline RUNX1 spectrum to also include lymphoid subtype malignancies, most commonly childhood T cell ALL (T-ALL), but also including lymphoma and, in some rare cases, B cell ALL (B-ALL) (Figure 1B) (6-8). Guideline recommendations for germline RUNX1 mutations in hematological malignancy predisposition by the WHO and others suggest mainstream germline testing is approaching (9). However, due to the range of phenotype and age of presentation, testing remains clinically challenging (10). Therefore, a complementary approach to the traditional clinical family

\section{Related Article: https://doi.org/10.1172/JCl147898}

Conflict of interest: The authors have declared that no conflict of interest exists.

history that may miss predisposition due to many factors, such as mild symptoms (e.g., low-normal platelets in germline RUNX1) and de novo mutations, includes the ability to identify germline predisposition variants in relevant tumor cohorts through unbiased genomic analysis. The increased use of clinical tumor next-generation sequencing (NGS) profiling panels provides both an opportunity and a challenge in this area. There is much discussion about how to identify and appropriately deal with germline findings from tumor sequencing, with published guideline suggestions for assessing myeloid predisposition, including potential germline $R U N X 1$ variants in this scenario as well as a recent position paper from the American College of Medical Genetics and Genomics (ACMG) encompassing guidelines across all tumor types $(11,12)$.

\section{Potential germline RUNX1 variants}

In this issue of the JCI, Li and Yang et al. (13) take an unbiased approach to annotating and testing potential germline RUNX1 variants. The researchers sequenced RUNX1 in remission samples from over 4800 cases of B-ALL and over 1300 cases of T-ALL from a large cohort of pediatric patients. Selecting for rare variants in remission samples, the authors identified putative germline variants for further assessment. Variants in both B-ALL and T-ALL cohorts differed in their characteristics. B-ALL variants were predominantly missense mutations concentrated toward the $\mathrm{C}$ terminus of the protein and outside of the RUNT homology domain (RHD), which is involved with DNA binding and where most known pathogenic RUNX1 missense variants are found (5). In contrast, the variants found in T-ALL were almost all stop-gain and frameshift variants and spread across the protein, consistent with potential loss-of-function variants. Functional assessment of variants is important 
A

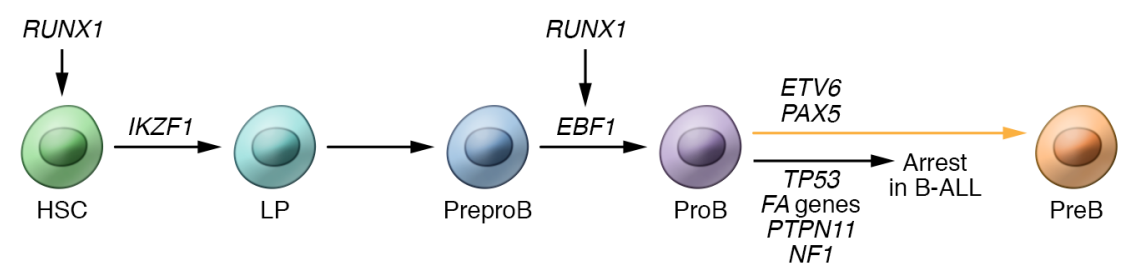

B

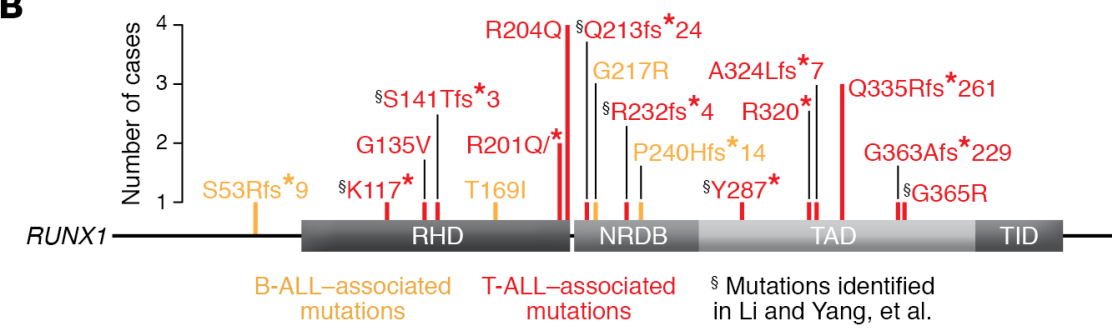

C

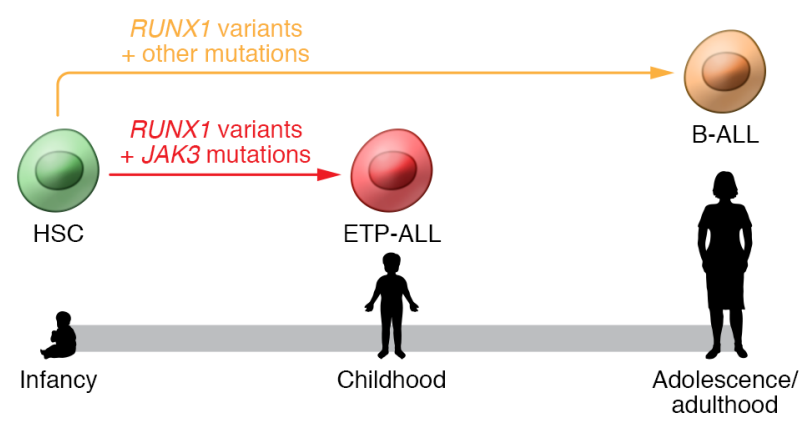

for the determination of pathogenicity of alleles and assists clinical classification. The ClinGen Myeloid Malignancy Variant Curation Expert Panel (MM-VCEP) recommendations for RUNX1 include a luciferase-based transactivation assay with a known RUNX1 target gene (14). Employing such an assay, the authors found that, consistent with the predicted effect on function, within the 20 B-ALL variants assessed, none had a reduction in transactivation activity, whereas in the 11 T-ALL-associated variants, six had reduced transactivation capacity. The combination of this functional data with other properties of these variants allowed four variants to be provisionally classified as pathogenic or likely pathogenic according to modified ACMG criteria (14). Given the rarity of germline RUNX1 mutations, unsurprisingly, these mutations are all novel in the germline RUNX1-FPD context, with two variants seen previously in a somatic context in the Catalogue of Somatic Mutations in Cancer (COSMIC) database. The commonality of the same variant occurring in both a somatic and germline context in tumors highlights a substantial challenge in identifying when RUNX1 variants are germline. Therefore, it is essential to confirm variants as germline using a nonhematological source of genetic material (e.g., hair follicle, cultured skin fibroblasts).

\section{The role of $R U N X 1$ variants in lymphoid leukemogenesis}

Out of the T-ALL-associated RUNX1 variants of interest identified by Li and Yang et al., two variants resulted in a loss of function due to nonsense and frameshift mutations in the RHD (13). Interestingly, four T-ALL-associated RUNX1 variants, in addition to the loss of transcriptional activity, appeared to have dominant-negative activity in assays of cellular context, adding support to previous data suggesting some germline RUNX1 variants have dominant-negative function (10). Further, functional assays in $\mathrm{CD} 34^{+}$cells performed on three variants with intact RHD showed phenotypes consistent with known myeloid
Figure 1. Model for B- and T-ALL predisposition. (A) Schematic of B cell development and normal function of known transcription factors involved in $\mathrm{B}-\mathrm{ALL}$ predisposition. RUNX1 is required for the emergence of developmental hematopoietic stem cells (HSCs), and it also regulates the induction of EBF1 essential for transition to pro-B cell. Germline variants of IKZF1, ETV6, and $P A X 5$ predominantly predispose patients to ALLs, while germline variants in PTPN11, NF1, TP53, and other genes involved in MAPK signaling and DNA damage are associated with both ALL and other malignancies. (B) RUNX1 germline variants reported in $\mathrm{Li}$ and Yang et al. (13) (§) and previously published work $\left(^{*}\right)$ associate with lymphoid malignancies B-ALL and T-ALL. (C) Damaging germline RUNX1 variants are observed in pediatric T-ALL patients and collaborate with somatic JAK3 mutations, whereas they are absent from pediatric B-ALL patients, with rare examples from other studies in which they are associated with older age of B-ALL onset, but do not have recurrent shared somatic variants. LP, lymphoid progenitor; PreproB,prepro B cell; proB, pro-B cell; preB, pre-B cell; FA, Fanconi anemia; NRDB, negative regulatory domain for DNA binding; TID, transcriptional inhibitory domain.

skewing that has also been observed in Runx1 deficiency models and RUNX1-FPD preclinical models $(10,15)$. Importantly, all variants resulted in decreased production of $\mathrm{CD} 41 \mathrm{a}^{+} \mathrm{CD} 42 \mathrm{~b}^{+}$megakaryocytes, a feature of RUNX1-FPD associated with mild thrombocytopenia in patients.

$\mathrm{Li}$, Yang, and coauthors next tested the specific role of the RUNX1 variants in leukemogenesis. First, gene-expression analysis of patient T-ALLs showed that leukemias with either germline or somatic RUNX1 mutations clustered closer to T-ALL with early $\mathrm{T}$ cell precursor (ETP) or near-ETP phenotype when compared with those with WT RUNX1. These results suggest that mutant RUNX1 can contribute to leukemia maintenance regardless of whether the mutation was inherited or acquired. Whole-genome sequencing analysis revealed an enrichment of missense JAK3 mutations in the T-ALL samples evaluated (83\%) (Figure 1C). Similarly, JAK3 mutations were also enriched in T-ALL cases with somatic RUNX1 (27\%) compared with RUNX1 WT T-ALL cases 
(less than $8 \%$ ). The authors used a murine transplantation model to test the effect of these two mutations in vivo. The recipients of JAK3-transfected hematopoietic stem and progenitor cells (HSPCs) had higher total peripheral WBC counts compared with controls. The RUNX1 variant recipients had lower WBC counts than controls, consistent with previous observations of mildly reduced reconstitution capacity of RUNX1 mutant HSPCs (15). Leukemia was observed only in recipients of JAK3- or JAK3/RUNX1-transfected HSPCs, arguing for the role of acquired JAK3 mutations in the context of RUNX1 variants for leukemogenesis. Interestingly, the leukemia in $J A K 3 / R U N X 1$ recipients compared with $J A K 3$-only recipients was more consistent with an ETP phenotype, further suggesting their collaboration is essential for driving this phenotypic expression of RUNX1 leukemia predisposition (13).

\section{Conclusions and future directions}

Where does the striking difference between RUNX1 variants in B-ALL and T-ALL stem from? Among lymphoid malignancies described in RUNX1-FPD to date, the predominance of those of $\mathrm{T}$ cell origin is clear. The role of RUNX1 in $\mathrm{T}$ cell biology has been described in $\mathrm{T}$ cell maturation and tolerance (16). Runx1-deficient mice have decreased common lymphoid progenitors (CLPs) and $\mathrm{CD}^{+} \mathrm{T}$ cells, which persist upon transplantation, suggesting a cell-autonomous effect (15). RUNX1 is commonly mutated in adult and pediatric T-ALL and promotes T-ALL growth (17); its loss leads to dysregulated oncogenes critical for maintenance of T-ALL (18), and it participates in a core transcriptional regulatory circuit regulated by TAL1 in T-ALL (19). Li and Yang et al. demonstrated that the RUNX1 variant negatively affected $\mathrm{T}$ cells and, when combined with the JAK3 mutation, led to leukemia. It is not yet known how its role in $\mathrm{T}$ cell development relates to T-ALL leukemogenesis.

RUNX1 is involved in various stages in normal B cell development (Figure 1A and ref. 20). In childhood B-ALL, RUNX1 most commonly associates with the proto-oncogene ETV6 as a fusion protein (ETV6RUNX1 fusion protein) (21). In the fusion complex, RUNX1 remains largely intact, possessing the RHD and the transcriptional activation domain (TAD). However, when ETV6-RUNX1 fusion protein is expressed in developing HSPCs, it blocks B cell development at the transition to CLPs (22). Studies in preclinical models suggest that ETV6-RUNX1 fusion has a specific role in childhood B-ALL, acting through its effect on developmental HSPCs, and it is largely absent in adult B-ALL (22). Given the effect on later developmental stages of B cells in RUNX1 deficiency, is it possible that $\mathrm{B}$ cell leukemogenesis requires a longer latency to acquire transforming mutations and thus would be unlikely to associate with childhood B-ALL? The study cohort in Li and Yang et al. (13) was relatively young (10.5 years plus or minus 5.7 years in AALL0232) (23). RUNX1 loss affects $B$ lineage priming at the stages of lymphoid-primed multipotential progenitor and CLPs, and a transforming secondary mutation in these short-lived, self-limiting populations is unlikely to occur in the first decade of life. Consequently, RUNX1 deficiency-related B-ALL may more likely be observed in adolescence or adulthood, when the transforming somatic mutation would occur in an earlier progenitor (Figure 1C). Asymptomatic occurrence of clonal hematopoiesis due to mutations in HSPCs has been described in RUNX1-FPD and is usually observed after the second decade of life (10). In fact, the four cases of B-ALL in RUNX1-FPD known to us were diagnosed in two adolescents and two adults in their 40s (6-8). The pathophysiologic link between RUNX1 loss and B-ALL leukemogenesis is yet to be established.

The work by Li and Yang et al. (13) highlights the importance of annotating and characterizing gene variants identified on widely used sequencing platforms in pediatric oncology today and builds on previous such efforts that have added TP53, $I K Z F 1$, and others to our repertoire of genes known to predispose to particular subtypes of ALL (1). Ongoing research efforts to build detailed clinical annotations of these rare disease cohorts with specific RUNX1 variants will contribute to our understanding of age of disease onset, treatmentrelated toxicities, and outcome of patients harboring these variants. Comprehensive clinical annotations will provide further opportunities to identify, monitor, and treat individuals at risk for ALL.

\section{Acknowledgments}

SA and ALB have received support from the RUNX1 Research Program and would also like to acknowledge the support from their colleagues and RUNX1-FPD patients and their families of previous and ongoing research efforts for this disorder.

Address correspondence to: Anna L. Brown, SA Pathology, Frome Road, Adelaide, South Australia 5070, Australia. Phone: 61.8.82223885; Email: Anna. Brown@sa.gov.au.

1. Bloom M, et al. Recent advances in genetic predisposition to pediatric acute lymphoblastic leukemia. Expert Rev Hematol. 2020;13(1):55-70.

2. Song WJ, et al. Haploinsufficiency of CBFA2 causes familial thrombocytopenia with propensity to develop acute myelogenous leukaemia. Nat Genet. 1999;23(2):166-175.

3. Bluteau $\mathrm{O}$, et al. A landscape of germline mutations in a cohort of inherited bone marrow failure patients. Blood. 2018;131(7):717-732.

4. Rio-Machin A, et al. The complex genetic landscape of familial MDS and AML reveals pathogenic germline variants. Nat Commun. 2020;11(1):1044.

5. Homan CC, et al. The RUNX1 Database (RUNX1db): establishment of an expert curated RUNX1 registry and genomics database as a public resource for familial platelet disorder with myeloid malignancy [published online July 8 , 2021]. Haematologica. https://doi.org/10.3324/ haematol.2021.278762.

6. Brown AL, et al. RUNX1-mutated families show phenotype heterogeneity and a somatic mutation profile unique to germline predisposed AML. Blood Adv. 2020;4(6):1131-1144.

7. Six KA, et al. B-cell acute lymphoblastic leukemia in patients with germline RUNX1. Blood Advances. In press.

8. Karki N, et al. Novel germline RUNX1 mutation associated with familial thrombocytopenia as well as B-acute lymphoblastic leukemia: a case report and review of the literature. Case Rep Oncol. 2021;14(1):439-245.

9. Arber DA, et al. The 2016 revision to the World Health Organization classification of myeloid neoplasms and acute leukemia. Blood. 2016;127(20):2391-2405.

10. Brown AL, et al. Secondary leukemia in patients with germline transcription factor mutations (RUNX1, GATA2, CEBPA). Blood. 2020;136(1):24-35.

11. Trottier AM, et al. Somatic mutation panels: time to clear their names. Cancer Genet. 2019;235-236:84-92.

12. Li MM, et al. Points to consider for reporting of germline variation in patients undergoing tumor testing: a statement of the American College of Medical Genetics and Genomics (ACMG). Genet Med. 2020;22(7):1142-1148.

13. Li Y, et al. Germline RUNX1 variation and predisposition to childhood acute lymphoblastic leukemia. J Clin Invest. 2021;131(17):e147898. 
14. Luo X, et al. ClinGen Myeloid Malignancy Variant Curation Expert Panel recommendations for germline RUNX1 variants. Blood Adv. 2019;3(20):2962-2979.

15. Growney JD, et al. Loss of Runx1 perturbs adult hematopoiesis and is associated with a myeloproliferative phenotype. Blood. 2005;106(2):494-504.

16. Hsu F-C, et al. An essential role for the transcription factor Runx1 in T cell maturation. Sci Rep. 2016;6:23533.

17. Jenkins CE, et al. RUNX1 promotes cell growth in human T-cell acute lymphoblastic leukemia by transcriptional regulation of key target genes. Exp Hematol. 2018;64:84-96.

18. Choi A, et al. RUNX1 is required for oncogenic Myb and Myc enhancer activity in T-cell acute lymphoblastic leukemia. Blood. 2017;130(15):1722-1733

19. Sanda T, et al. Core transcriptional regulatory circuit controlled by the TAL1 complex in human T cell acute lymphoblastic leukemia. Cancer Cell. 2012;22(2):209-221.

20. Niebuhr B, et al. Runx1 is essential at two stages of early murine B-cell development. Blood.
2013;122(3):413-423.

21. Romana SP, et al. The t(12;21) of acute lymphoblastic leukemia results in a tel-AML1 gene fusion. Blood. 1995;85(12):3662-3670.

22. Schindler JW, et al. TEL-AML1 corrupts hematopoietic stem cells to persist in the bone marrow and initiate leukemia. Cell Stem Cell. 2009;5(1):43-53.

23. Borowitz MJ, et al. Prognostic significance of minimal residual disease in high risk B-ALL: a report from Children's Oncology Group study AALL0232. Blood. 2015;126(8):964-971. 